\title{
Constraining the progenitor evolution of GW 150914
}

\author{
Jorick S. Vink ${ }^{1}$ \\ ${ }^{1}$ Armagh Observatory and Planetarium, BT61 9DG Armagh, College Hill, Northern Ireland \\ email: jorick.vink@armagh.ac.uk
}

\begin{abstract}
One of the largest surprises from the LIGO results regarding the first gravitational wave detection (GW 150914) was the fact the black holes (BHs) were "heavy", of order 30 - 40 $M_{\odot}$. The most promising explanation for this obesity is that the BH-BH merger occurred at low metallicity $(Z)$ : when the iron $(\mathrm{Fe})$ contents is lower this is expected to result in weaker mass loss during the Wolf-Rayet (WR) phase. We therefore critically evaluate the claims for the reasons of heavy BHs as a function of $Z$ in the literature. Furthermore, weaker stellar winds might lead to more rapid stellar rotation, allowing $\mathrm{WR}$ and $\mathrm{BH}$ progenitor evolution in a chemically homogeneous manner. However, there is as yet no empirical evidence for more rapid rotation amongst WR stars in the low $Z$ environment of the Magellanic Clouds. Due to the intrinsic challenge of determining WR rotation rates from emission lines, the most promising avenue to constrain rotation-rate distributions amongst various WR subgroups is through the utilisation of their emission lines in polarised light. We thus provide an overview of linear spectro-polarimetry observations of both single and binary WRs in the Galaxy, as well as the Large and Small Magellanic Clouds, at $50 \%$ and $20 \%$ of solar $Z$, respectively. Initial results suggest that the route of chemically homogeneous evolution (CHE) through stellar rotation is challenging, whilst the alternative of a post-LBV or common envelope evolution is more likely.
\end{abstract}

Keywords. gravitational waves, polarization, stars: early-type, stars: evolution, stars: mass loss, stars: rotation, stars: winds, outflows, stars: Wolf-Rayet

\section{Introduction}

One of the main surprises regarding the first gravitational wave detections by LIGO concerning the physical merging of 2 black holes (BHs) was the fact that the masses inferred for these BHs were very heavy - of order $30-40 M_{\odot}$ (Abbott et al. 2016). Within our own Galactic environment the maximum black hole mass is thought to be of order $10 M_{\odot}$ (Belczynski et al. 2010).

For this reason there are 2 possible solutions to the problem. Either the GW150914 event took place in an environment that was low metallicity, reducing the amount of mass loss during stellar evolution, or the initial stars started off with very high masses. Even if there had been relatively little mass loss, the initial masses must have been higher than $40 M_{\odot}$, relating to a stellar mass regime where winds are a critical ingredient for massive-star evolution (e.g. Higgins \& Vink 2018). For very massive stars (VMS), defined with masses over $\simeq 100 M_{\odot}$ (Vink et al. 2015), stellar winds completely dominate their evolution and fate (Woosley \& Heger 2015; Hirschi 2015).

In this contribution, we constrain the physics and evolution of the progenitor of GW150914 in terms of the geometry and the amount of the mass loss from Wolf-Rayet (WR) stars, which are thought to be the direct progenitors of BHs. 


\section{Stellar winds at low metallicity}

Whilst it has been known for decades that stellar winds during the initial O star phase depend on metallicity $Z$ (Abbott et al. 1982; Kudritzki et al. 1987; Vink et al. 2001), the realisation that WR stars also depend on the iron (Fe) contents of the host galaxy is rather more recent (Vink \& de Koter 2005; Hainich et al. 2015). Until 2005, most stellar evolution modellers assumed that the host metallicity (Fe) was so low in terms of stellar abundance when compared to the self-enriched carbon during the WC phase that mass loss was assumed to be independent of host galaxy metallicity.

Using an established Monte Carlo method, Vink \& de Koter (2005) showed that WR mass-loss rates depend on the host galaxy metallicity ( $\mathrm{Fe}$ ) after all. Figure 1 shows the predicted WR mass-loss versus $Z$ dependence for both nitrogen-rich WN stars (in black solid) and the slightly shallower $Z$ dependence for carbon-rich WC stars (in dashed green). Note that the self-enriched materials are not taken into account in the definition of $Z$ on the x-axis. In more recent times, Vink (2017) confirmed the theoretical $Z_{\mathrm{Fe}}$ dependence for optically thin stripped helium stars using a dynamically consistent version of the Monte Carlo method (Müller \& Vink 2008).

A direct consequence of including $Z$ dependent mass-loss rates of WR stars into massive star evolution models was the prediction of more massive BHs (Eldridge \& Vink 2006). Figure 2 shows the expected maximum $\mathrm{BH}$ mass at a given host galaxy metallicity from a single star population synthesis by Belczynski et al. (2010). The dashed red line indicates the older view that independent of host galaxy $Z$ the maximum BH mass would not exceed $\sim 10-20 M_{\odot}$, as the WR mass-loss rates were thought to be due to self-enrichment by carbon and oxygen. Only when including the newer Vink \& de Koter (2005) WR Fe dependent mass-loss rates one may expect to find heavier BHs at lower metallicities.

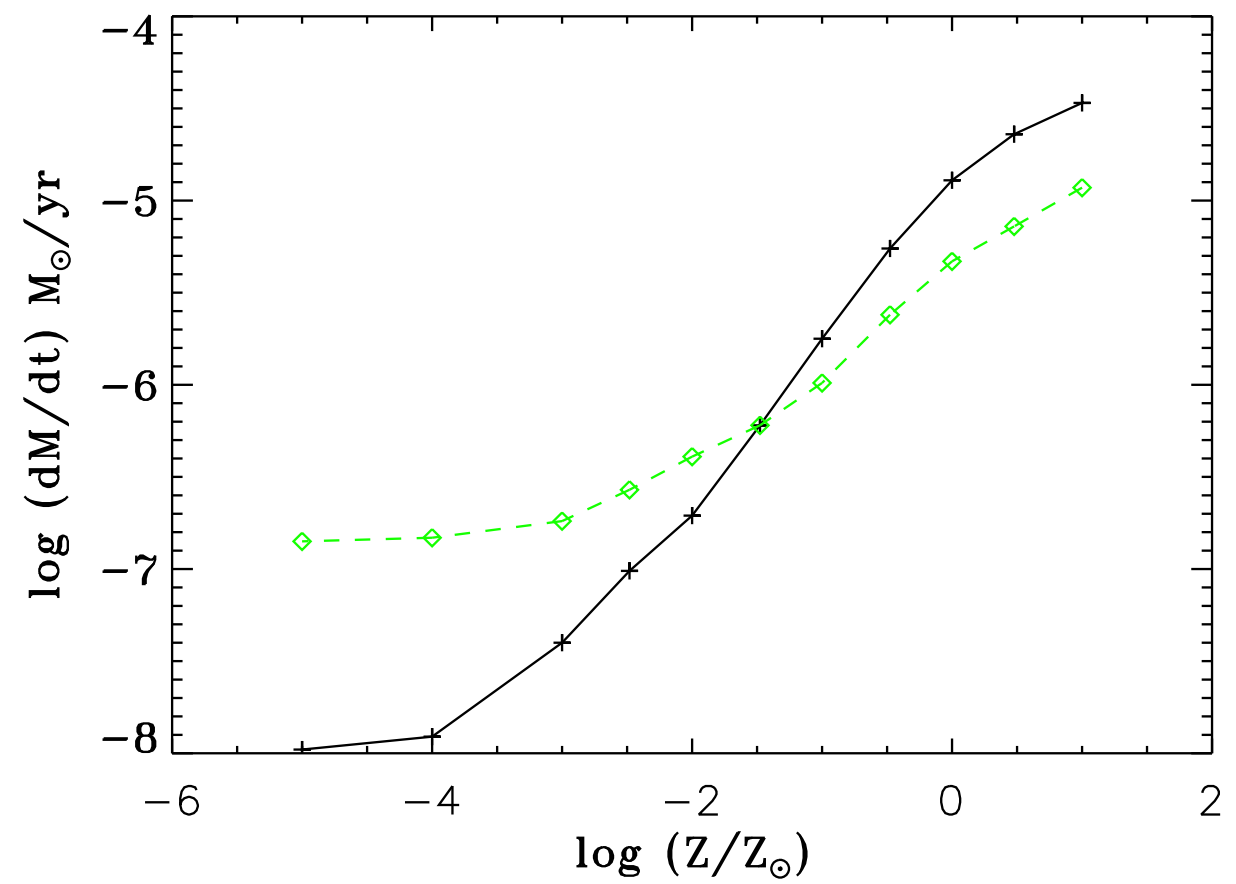

Figure 1. Predicted mass-loss rates of WR stars versus host galaxy metallicity. The black solid line indicates the steeper dependence for the nitrogen-rioch WN stars, whilst the green dashed line indicates the shallower slope for the carbon-rich WC stars. From Vink \& de Koter (2005). 
Exactly the same plot was also included in the astrophysical interpretation paper of GW 150914 by the LIGO consortium (Abbott et al. 2016) but, confusingly, the older and newer expectations of WR mass loss versus $Z$ implementations were here referred to as 'stronger' and 'weaker' stellar winds. That is not correct, as absolute values of the mass-loss rates have not changed. Instead, it is the weaker mass loss at lower $Z$ that is the key physics explaining the heavier BHs at low $Z$. At the same time, there have not been any substantial changes at solar $Z$, as can be seen at the intersection of the 2 curves on the left-hand side of Figure 2.

Simply reading off the y-axis of Figure 2 for a $40 M_{\odot}$ maximum BH mass, immediately leads to the conclusion that the chemical environment of the GW150914 progenitor should have been $1 / 10 Z_{\odot}$ or less. Detailed single star or binary evolution does not appear to be particularly relevant for making this particular inference. Instead, given the intrinsically low $Z$, GW150914-type events can teach us interesting physics about winds and stellar evolution at low $Z$.

\section{Interplay between rotation and winds}

In order to not only predict compact object masses, but also their spin, it is important to consider the theory of stellar rotation and winds. The first aspect is that the relation

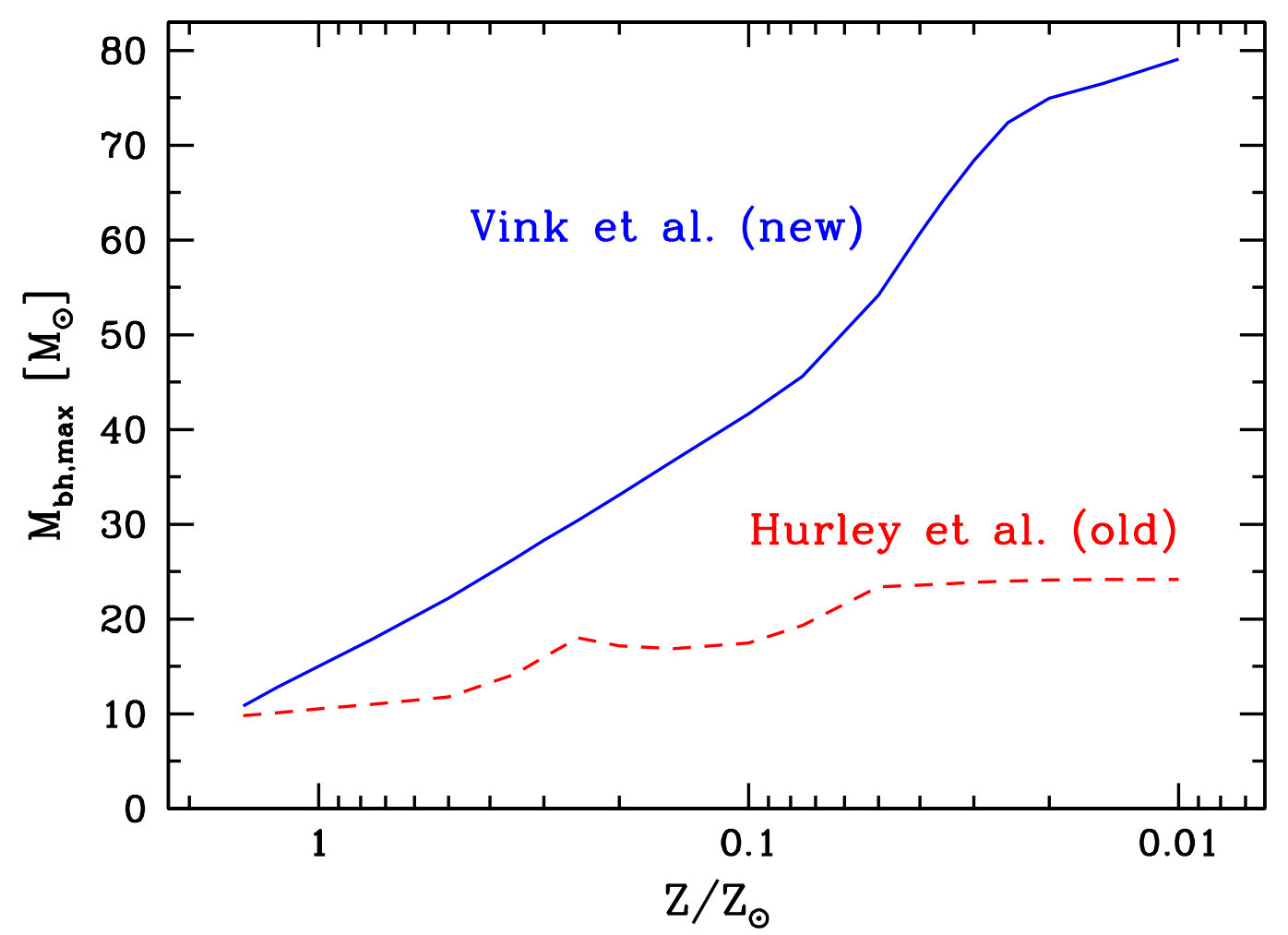

Figure 2. Predicted maximum BH mass versus metallicity. The red dashed line shows the almost $Z$ independent maximum BH mass when including the situation around the year 2000 (Hurley et al. 2000), whilst including the $Z$ dependence of not only the Vink et al. (2001) O-type stars, but also the WR $Z$ dependent winds of Vink \& de Koter (2005) leads to a strong sensitivity of maximum BH mass on $Z$, as indicated by the blue solid line. From Belczynski et al. (2010). The same plot with incorrect labels was included in Abbott et al. (2016). 
can work in both directions: stellar winds may remove angular momentum, thus braking the star, even in the absence of a magnetic field (e.g. Langer 2012), but reversely it has been argued that stellar rotation may increase the overall mass-loss rate (e.g. Maeder \& Meynet 2000). In more recent times, 2D dynamical calculations by Müller \& Vink (2014) showed there are cases where the overall mass-loss rate may actually decrease with respect to their non-rotating counterparts.

The third relevant aspect is to consider the geometry of rotating winds: do we expect equatorial enhancement (which may remove angular momentum very efficiently) or polar winds? Let us briefly review the key physical ingredients. Friend \& Abbott (1986) argued that as a result of a lower effective gravity from a rotating star, mass loss from the equator would be more efficient than from the pole. Later, Cranmer \& Owocki (1995) found that due to the Von Zeipel gravity darkening the pole would be brighter and thus mass would preferentially be lost from the pole instead. Alternatively, taking both these competing effects into account, for certain temperatures around the bi-stability jump temperature, mass would predominately be lost from the equator after all (Pelupessy et al. 2000). It is clear that the situation regarding the geometry is complex, and that theory needs guidance from observations in order to make progress.

\section{Testing stellar rotation with polarimetry}

Until astronomers are able to spatially resolve the innermost radii of hot massive stars, linear spectropolarimetry is the only tool available to dissect geometry of stellar winds. The technique was already applied in the 1970s for classical Be stars, and was later also applied to young pre-main sequence Herbig Ae/Be and T Tauri stars to understand accretion disks. One of the key strengths of spectropolarimetry is that there is no depen-

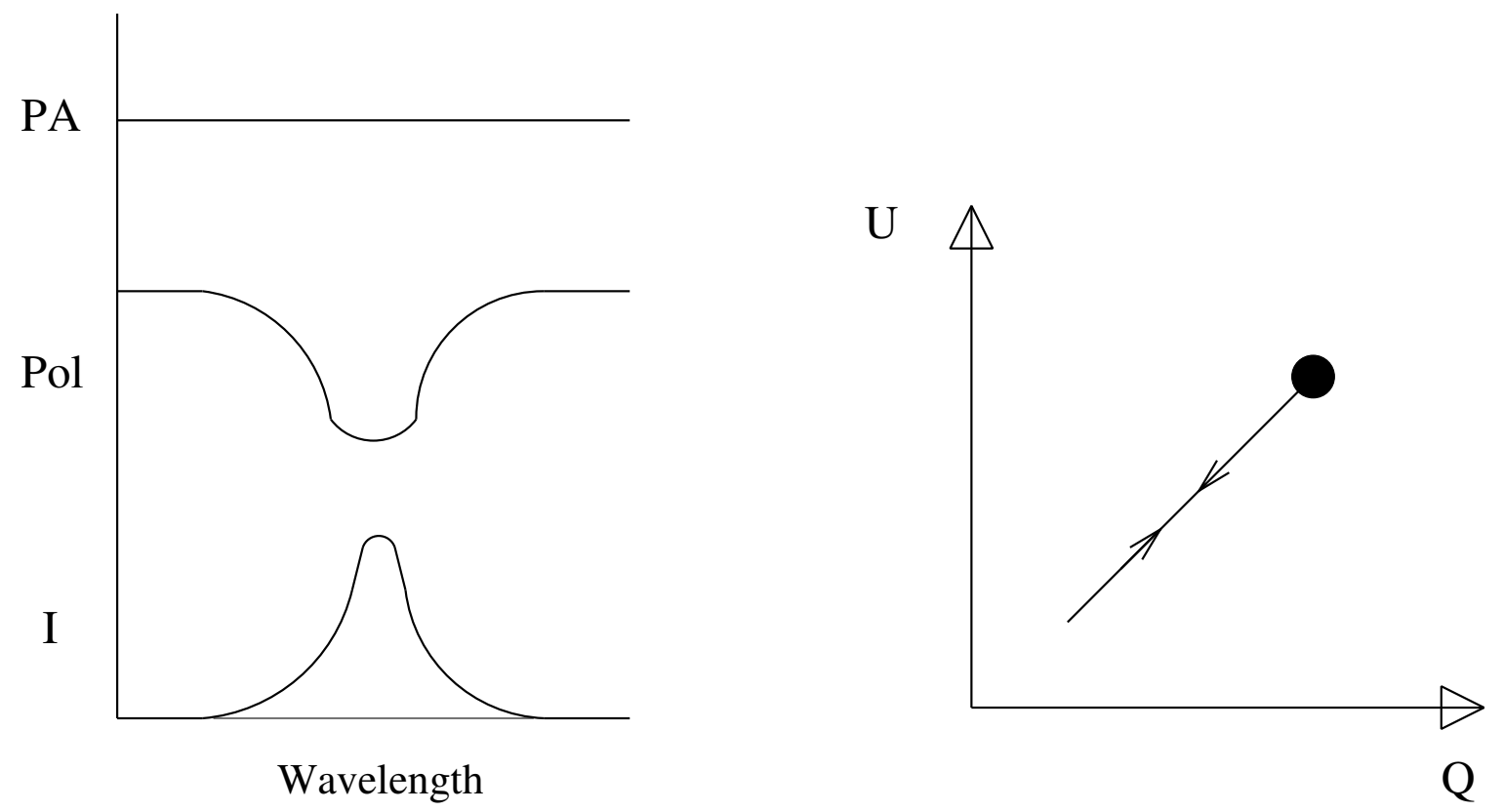

Figure 3. Polarization triplot (on the left-hand side) and the Stokes QU plane (right-hand side). For an emission line (lower panel triplot) one expects a 'depolarization' in the middle panel of the triplot if the innermost geometry is flattened. For a simple disk , the position angle (PA) of the disk remains constant, and the PA can also be read off from the QU plane (right-hand side). 
dence on any dust particles between the star under consideration and the observer (due to interstellar polarization).

Figure 3 shows the expectations from a disk around a star in the polarization triplot (on the left-hand side) and the Stokes QU plane. For an emission line (lower panel triplot), one only expects to see a 'depolarization' in the middle panel of the triplot if the innermost geometry is significantly flattened, i.e. disk-like. For a simple disk, the position angle (PA) remains constant, and the PA can also be determined from the QU plane (see the right-hand side).

Given the expected lower mass-loss rates of WR stars at lower $Z$, one might perhaps expect disks to be more prevalent at lower $Z$ than in our Milky Way. Indeed many B-type stars in the SMC seem to be Be stars (e.g. Castro et al. 2018). However, in our recent VLT-FORS polarisation study of large samples of WR stars in the low $Z$ environments of the Magallanic Clouds (39 in the LMC; all $12 \mathrm{WRs}$ in the SMC) we found the incidence of depolarisation 'line effects' (Fig. 3) to be indistinguishable from those in the Milky Way (Vink \& Harries 2017).

This appears to be quite a revelation for stellar modellers attempting to explain the evolution of GW 150914 with physics related to rapid rotation, such as the rotationallyinduced chemically homogeneous evolution (CHE), as we have basically no empirical evidence that WR stars at low metallicity rotate any faster than those in the Milky Way.

\section{Remarks on evolutionary scenarios}

The results of the VLT study of Vink \& Harries (2017) suggest that WR stars in low $Z$ environments do not rotate any faster than at high $Z$. What does this imply for the subsequent evolution of WR stars into $\mathrm{BHs}$, and how can this information be used to constrain the evolution towards the WR phase?

Independent of any subtle binary evolution effects that will undoubtedly come into play when we wish to explain the compact object mass spectrum, we can already learn some lessons regarding the physics of either component in the merging $\mathrm{BH}$ binary progenitor.

First of all, the concept of CHE for the formation of WR stars at low $Z$ due to rotationally-induced CHE (Yoon \& Langer 2005) is challenging to entertain given the lack of evidence for WR rotation at low $Z$. This would therefore also make it harder for this evolutionary pathway to be relevant for the very specific event of GW150914 (e.g. Mandel \& de Mink 2016; Marchant et al. 2016), unless such events are evolutionary unrelated to ordinary single star and binary evolution at high and low $Z$, as our VLT sample contained both single WRs and binaries, and no difference in the line-effect frequency was noted.

Vink et al. (2011) argued that the most likely explanation for the fact that only a 10$20 \%$ sub-population of WR stars is found to rotate is related to a more classical post-LBV like evolutionary channel. In the context of binary evolution we might translate this to common-envelope evolution (Belczynski et al. 2016), although the interesting discussion on CHE should certainly continue!

\section{References}

Abbott, D. C. 1982, ApJ, 259, 282

Abbott, B. P., Abbott, R., Abbott, T. D., et al. 2016, ApJ, 818, L22

Belczynski, K., Bulik, T., Fryer, C. L., et al. 2010, ApJ, 714, 1217

Belczynski, K., Holz, D. E., Bulik, T., \& O'Shaughnessy, R. 2016, Nature, 534, 512

Castro, N., Oey, M. S., Fossati, L., \& Langer, N. 2018, arXiv:1810.04682

Cranmer, S. R., \& Owocki, S. P. 1995, ApJ, 440, 308 
Eldridge, J. J., \& Vink, J. S. 2006, A\&A, 452, 295

Friend, D. B., \& Abbott, D. C. 1986, ApJ, 311, 701

Hainich, R., Pasemann, D., Todt, H., et al. 2015, A\&A, 581, A21

Higgins, E. R. \& Vink, J. S., 2018, A\&A submitted

Hirschi, R. 2015, Very Massive Stars in the Local Universe, 412, 157

Hurley, J. R., Pols, O. R., \& Tout, C. A. 2000, MNRAS, 315, 543

Kudritzki, R. P., Pauldrach, A., \& Puls, J. 1987, A\&A, 173, 293

Langer, N. 2012, ARA\&A, 50, 107

Maeder, A., \& Meynet, G. 2000, A\&A, 361, 159

Mandel, I., \& de Mink, S. E. 2016, MNRAS, 458, 2634

Marchant, P., Langer, N., Podsiadlowski, P., Tauris, T. M., \& Moriya, T. J. 2016, A\&A, 588, A50

Müller, P. E., \& Vink, J. S. 2008, A\&A, 492, 493

Müller, P. E., \& Vink, J. S. 2014, A\&A, 564, A57

Pelupessy, I., Lamers, H. J. G. L. M., \& Vink, J. S. 2000, A\&A, 359, 695

Vink, J. S. 2017, A\&A, 607, L8

Vink, J. S., \& de Koter, A. 2005, A\&A, 442, 587

Vink, J. S., \& Harries, T. J. 2017, A\&A, 603, A120

Vink, J. S., de Koter, A., \& Lamers, H. J. G. L. M. 2001, A\&A, 369, 574

Vink, J. S., Gräfener, G., \& Harries, T. J. 2011, A\&A, 536, L10

Vink, J. S., Heger, A., Krumholz, M. R., et al. 2015, Highlights of Astronomy, 16, 51

Woosley, S. E., \& Heger, A. 2015, Very Massive Stars in the Local Universe, 412, 199

Yoon, S.-C., \& Langer, N. 2005, A\&A, 443, 643 